\title{
Issue highlights
}

\author{
Dr. Sujit Bhattacharya Ph. D \\ Editor-in-Chief, Journal of Scientometric Research, Senior Principal Scientist (NISTADS) \\ Dr. K.S. Krishnan Marg, Pusa Campus, New Delhi-110012, INDIA
}

The inaugural issue is expansive in its scope and covers a broad range of conceptual domains within scientometrics. The issue contains one review paper, six research articles, one research note and one webliography. The papers can be delineated under the following major subheads: conceptual, development of tools \& techniques, scientometric analysis of research areas of socio-economic importance, scientometric analysis covering new sources of supply, country study, and information resource management. We hope you will find this first inaugural issue useful and interesting. Brief highlights of each article are provided below.

The review paper by Ronald Kostoff underscores the potentiality and various approaches of text mining in science and technology. The review is in two parts to be published in two issues of this journal; Part I published in this issue focuses on text mining to the technical literature of interest to identify patterns to increase understanding of the topical matter, mainly its non-citation components. The review shows how tools and techniques that fall within the ambit of text mining have been applied in Scientometrics data sets, how the techniques have been refined to the problematic being studied, and qualitative distinctions between the different text mining approaches. The review illustrates the above aspects through author's extensive research in this area. This review along with part II will provide scholars and new entrants to the various text mining possibilities for enriching data analytics.

Ronald Rousseau and Fred Y. Ye paper revisits the indicators constructed from two basic bibliometric entitiespublications and citations. Through elegant mathematical

\section{${ }^{*}$ Corresponding author.}

Dr. Sujit Bhattacharya Ph. D

Editor-in-Chief, Journal of Scientometric Research

Senior Principal Scientist (NISTADS)

Dr. KS Krishnan Marg, Pusa Campus, New Delhi-110012, INDIA

Landline: +91-11-25843024

Mobile: $+91-9999020157$

E-mail: editor@jscires.org | www.jscires.org

DOI: 10.5530/jscires.2012.1.4 constructs the properties of indicators and the dynamic relationship between publications and citations are explored. The article introduces the basic independence axiom for an indicator and 'h-critical publication' which further qualifies the popular ' $h$ ' indicator. Bibliometric indicators are nowadays liberally used and increasingly being relied upon in evaluation and competitive judgment among others. However, in many cases they are wrongly used leading to erroneous interpretations which questions the tools and techniques. This paper is important and particularly of high relevance in the present context as it provides conceptual clarity to the usage of bibliometric indicators.

Loet leydesdorff and Ulrike Felt draw attention to the extensive bibliometric analysis now possible for two new sources of publications-book and book chapters as they are now indexed in databases. This study is among the first few research work in developing an analytical framework for these two sources. It opens up to the exciting possibility of expanding the scope of bibliometrics which can provide further insights to the knowledge landscape.

Christian Gumpenberger, Ambros Wernisch and Juan Gorraiz examine contemporary issue of high relevance: journal evaluation for journal acquisition management in a library. Traditionally journal evaluation has been one of the major motivations behind bibliometric studies, but with changing dynamics the contours of the problem has changed significantly. Changing dynamics of journal publishing, creation of cartels and highly skewed pricing and price fluctuations provides challenge to library journal acquisition. These issues find excellent reflections in the problem definitions and analysis undertaken by the authors.

It makes better appreciation and relevance of bibliometric findings when plausible causality issues are examined. Paper by Jacqueline Leta underscores this aspect in her examination of recent trends in Brazilian science. Examining one of the key input factors-student outturn in different fields in Brazil, the paper provides a more insightful understanding of research outputs (trends and structural 
characteristics). One of the key areas of scientometric studies has been examination of national research trends of different countries. This paper draws attention to one of the key plausible factor-human resource and S\&T personal trend. This type of analysis makes scientometrics more relevant as policy input.

Gangan Prathap and Loet leydesdroff draw attention to new quality measures derived from citations for performance measurement. Drawing from author's extensive research in this area they show how the new measures can overcome some of the problematic associated with earlier approaches. They apply their analytical approach to assess the progress of academic research performance of the world over the decade from 2000 to 2010 . The study we posit will influence and generate further critical introspection in this contemporary area of research.

Newspapers play an important role in creating public perceptions/opinions and thus systematic analysis of its content over a period of time is an important reflection of the same. The science-society relationship and policy directions are also shaped to a great extent by newspapers. Bharvi Dutt and K.C. Garg analyse biomedical research coverage in the Indian English language newspapers using statistical and content analysis approach to underscore nature and trend of bio-medical coverage. Their analysis points out to the skewed nature of coverage more directed towards lifestyle related health issues neglecting issues of pressing health concerns and explore causality behind this skewed representation.

Adarsh Bala and B.M. Gupta investigate research efforts in measles by analyzing research papers covering this disease over a period of ten years (2001-10). The paper indentifies the different facets of measles research such as loci of research action, research linkages, and trends. This paper is timely keeping in view the importance of this in assessment of research in an area of pressing health concern and also of the fact that inspite of this only a few papers have investigated research trends in this area.

Divya Srivastava and Renu Bahadur primarily address two major issues - the increasing important role websites can play in health issues (taking the case study of Geriatrics), and various attributes that can benchmark web-sites. The paper also highlights interesting aspects of websites longevity, linkage, visibility, etc. This paper is highly relevant in the present context as wider access possible through e-resources is leading to the emergence of this as a new communication mode of scholarly communication.

Keeping in view the ever increasing resources available in the web, it becomes a challenging exercise to delineate key materials in each intellectual domains and segments within it. Scientometrics has also been enriched by the availability of e-resources: open access and proprietary research materials. Scientometrics is a broad domain in itself and comprises many core sub-domains and also has connections to intellectual domains covering STS, Innovation studies, Policy research, etc. Webliography of Science, technology and innovation indicators by Anup Das, Praveen Arora and Sujit Bhattacharya brings together institutes, databases, and key reports that cover STI (Science, Technology, and Innovation) indicators. The salient features of these key reports and links to the full resources are provided. Along with identifying some key global and national reports, the webliography also identifies some useful measurement toolkits. The present webliography is a useful first step in organizing the scholarly e-resources in Scientometrics. 